DAMTP-95-2

hep-th/9501079

\title{
Lattice quantization of Yangian charges
}

\author{
N. J. MacKay*† \\ Dept of Applied Maths and Theoretical Physics, \\ Cambridge University, \\ Cambridge, CB3 9EW, UK
}

\begin{abstract}
By placing theories with Yangian charges on the lattice in the analogue of the St Petersburg school's approach to the sine-Gordon system, we exhibit the Yangian structure of the auxiliary algebra, and explain how the two Yangians are related.
\end{abstract}

\section{Introduction}

In 1990 Bernard ${ }^{[1]}$, building on work by Lüscher ${ }^{[2]}$, showed that the algebra of non-local charges in 1+1-D quantum field theories with curvature-free conserved currents is precisely Drinfeld's Yangian algebra ${ }^{[3]}$. The naive definition of the first non-local charge is divergent, and must be regularized; Bernard did this on the continuum. The coproduct gives the action of the charges on asymptotic states, and conservation of the charges allows one, in principle, to determine the scattering matrix. The classical $\hbar \rightarrow 0$ limit ${ }^{[4]}$ of the charge algebra retains the Yangian structure in both the Poisson brackets and (a classical definition of) the coproduct (see appendix). No spectral parameter or auxiliary algebra appears in this procedure, and its use was therefore presented as an alternative to the quantum inverse scattering method.

*Supported by a PPARC fellowship

${ }^{\dagger}$ n.j.mackay@damtp.cambridge.ac.uk 
Recall now how Drinfeld introduced the Yangian, as the unique quantum deformation of a Lie algebra consistent with a Casimir-like classical $r$-matrix. This Yangian is the quantum version of the auxiliary algebraic structure introduced when integrability of a classical equation (in this case, conservation and curvature-freedom of the current) is expressed through a Lax pair, which involves a spectral parameter.

Drinfeld's mathematical intuition may be contrasted with the physical intuition whereby Kulish and Reshetikhin ${ }^{[5]}$ discovered the quantum deformation of $s u(2)$. The quantum inverse scattering method (QISM) of the St Petersburg school takes the classical Lax system and its monodromy matrix, which diverges upon quantization, and regularizes the quantum system by placing it on a spatial lattice, defining the spatial lattice Lax operator as the quantization of the monodromy matrix over one lattice step, which is finite. Making the quantum analogue of the classical $r$-matrix relation soluble, however, may require that the ('auxiliary') algebraic structure of the classical Lax pair be deformed. For sine-Gordon theory the classical $s u(2)$ algebra becomes what is now known as $s u_{q}(2)$.

It is therefore interesting to see whether we can relate the Yangian charges, which act on the 'quantum' space of QISM terminology, to the Yangian of the 'auxiliary' space, by quantizing on the lattice the Lax system of models with Yangian charges. We find that what is apparently a loop algebra acting on the auxiliary space must indeed be replaced by a Yangian, and in doing so place the Yangian on the same footing as $s u_{q}(2)$ in terms of the intuitions and historical development of QISM.

\section{Auxiliary Yangian structure}

Consider a classical current which is conserved,

$$
\partial^{\mu} j_{\mu}(x, t)=0
$$

curvature-free,

$$
\partial_{\mu} j_{\nu}-\partial_{\nu} j_{\mu}+\left[j_{\mu}, j_{\nu}\right]=0
$$

and Lie algebra valued,

$$
j_{\mu}(x, t)=t^{a} j_{\mu}^{a}(x, t)
$$


(where the $t^{a}$ generate a Lie algebra $\mathcal{A},\left[t^{a}, t^{b}\right]=f^{a b c} t^{c}$ ). These conditions are equivalent to the vanishing of the curvature

$$
\left[\partial_{0}+L_{0}, \partial_{1}+L_{1}\right]=0
$$

of the Lax pair

$$
L_{\mu}(x, t ; u)=\frac{1}{1-u^{2}}\left(j_{\mu}(x, t)+u \epsilon_{\mu}^{\nu} j_{\nu}(x, t)\right)
$$

(with convention $\eta_{00}=-\eta_{11}=1$ ) and thus to the solubility of $\left(\partial_{1}+L_{1}\right) T=0=\left(\partial_{0}+L_{0}\right) T$. The equation in the spatial derivative,

$$
\left(\partial_{x}+L_{1}(x ; u)\right) T(x, y ; u)=0
$$

has the formal solution

$$
T(x, y ; u)=\mathbf{P} \exp \left(-\int_{y}^{x} L_{1}(\xi ; u) d \xi\right)
$$

where $\mathbf{P}$ denotes path ordering on a path at a fixed time, and $t$ is a suppressed label. The integrability of the system is expressed by

$$
\left\{T(u) \stackrel{\otimes}{,} T\left(u^{\prime}\right)\right\}=\left[r\left(u, u^{\prime}\right), T(u) \otimes T\left(u^{\prime}\right)\right]
$$

where

$$
T(u)=T(\infty,-\infty ; u), \quad r\left(u, u^{\prime}\right)=\frac{1}{u^{\prime}-u} t^{a} \otimes t^{a},
$$

and the tensor product is in the auxiliary space; taking the trace of this relation gives an infinity of charges in involution. The passage to the quantum theory is beset by problems due to divergences in (1) and (2) which arise from products of quantum operators valued at the same point. The solution of the St Petersburg school ${ }^{[6]}$ is to put the theory on a lattice and define the quantum theory by its lattice transfer matrix, which is fixed by the requirements of integrability, i.e. that

$$
R\left(u, u^{\prime}\right) L_{N}^{1}(u) L_{N}^{2}\left(u^{\prime}\right)=L_{N}^{2}\left(u^{\prime}\right) L_{N}^{1}(u) R\left(u, u^{\prime}\right)
$$

be soluble, where $L_{N}$ is the one-step lattice transfer matrix and

$$
L_{N}^{1}(u)=L_{N}(u) \otimes 1 \quad L_{N}^{2}\left(u^{\prime}\right)=1 \otimes L_{N}\left(u^{\prime}\right)
$$

and of

$$
R\left(u, u^{\prime}\right) T^{1}(u) T^{2}\left(u^{\prime}\right)=T^{2}\left(u^{\prime}\right) T^{1}(u) R\left(u, u^{\prime}\right)
$$


having the correct classical limit (3) (where $T$ is the full lattice transfer matrix, $\prod_{N=-\infty}^{\infty} L_{N}$ ).

The natural ansatz ${ }^{[7,8]}$ for $L_{N}$ is to set

$$
L_{N} \equiv T((N-1 / 2) \Delta,(N+1 / 2) \Delta ; u)=\mathbf{P} \exp \left(-\int_{\left(N-\frac{1}{2}\right) \Delta}^{\left(N+\frac{1}{2}\right) \Delta} L_{1}(\xi ; u) d \xi\right)
$$

where the lattice points are $x_{N}=N \Delta$. We can then calculate $L_{N}$ using the canonical quantization of $L_{1}(x ; u)$, and find, writing the operator products as the sum of a regular part and a commutator, that it is finite. Using light-cone components of the current, $j_{ \pm} \equiv \frac{1}{2}\left(j_{0} \pm j_{1}\right)$, we take the commutation relations of the lattice currents

$$
j_{N \pm} \equiv \int_{\left(n-\frac{1}{2}\right) \Delta}^{\left(n+\frac{1}{2}\right) \Delta} j_{ \pm}(\xi) d \xi
$$

to be

$$
\left[j_{N \pm}, j_{M \pm}\right]=-i \hbar g \delta_{N M} j_{N \pm}-\frac{i k \Delta}{\lambda} \delta_{N M} C_{2}
$$

where $k$ is an unknown constant with the dimensions of energy, $\lambda$ a coupling constant, $C_{2}=t^{a} t^{a}$ and $\delta^{a d} g=f^{a b c} f^{d c b}$. (The classical limit has to be very carefully taken, with the space component of the current rescaled by a factor of $\hbar / \Delta$ in the way suggested by Faddeev and Reshetikhin ${ }^{[9]}$, in order to get the Poisson brackets of the principal chiral model $\mathcal{L}=\lambda^{-1} \int \operatorname{Tr}\left(\partial g^{-1} \partial g\right)$.) For the moment we shall set $k=0$, and describe what happens for non-zero $k$ later.

We then obtain

$$
L_{N}(u)=1+\sum_{n=0}^{\infty}\left(\left(\frac{1}{1-u}\right)^{n+1} j_{N-}^{a}+\left(\frac{-1}{1+u}\right)^{n+1} j_{N+}^{a}\right)(-1)^{n} t_{n}^{a}+\mathcal{O}\left(\epsilon^{2}\right)
$$

where

$$
t_{n}^{a}=(\epsilon g)^{n} t_{a}, \quad \epsilon=\frac{i \hbar}{2} .
$$

(Note that our approach has been to treat $\epsilon g$ as a loop variable, independent of $\epsilon$; none of its powers have been absorbed into the $\mathcal{O}\left(\epsilon^{2}\right)$ terms.) We shall see in the next section that the currents, when summed in the monodromy matrix, give charges which form a Yangian (acting on the quantum space). Similarly the $t_{n}^{a}$, we shall find when we work out $L_{N}^{1} L_{N}^{2}$, must be deformed from loop algebra generators into the generators of another Yangian (acting on the auxiliary space), and it follows (upon expanding and re-summing the $(1 \pm u)^{-n-1}$ terms) that

$$
L_{N}(u)=1-T_{u} \sum_{n=0}^{\infty} t_{n}^{a} j_{N \mu}^{a}+\mathcal{O}\left(\epsilon^{2}\right)
$$


where $\mu=0$ for $n$ odd and $\mu=1$ for $n$ even, and $T_{u}$ acts on $t_{n}^{a}$ according to (Y6). Working out $L_{N}^{1} L_{N}^{2}$ in the same way we find

$$
L_{N}^{1}(u) L_{N}^{2}\left(u^{\prime}\right)=1 \otimes 1-T_{u} \otimes T_{u^{\prime}} \sum_{n=0}^{\infty} \Delta\left(t_{n}^{a}\right) j_{N \mu}^{a}+\mathcal{O}\left(\epsilon^{2}\right)
$$

(with $\Delta\left(t_{n}^{a}\right)$ given by $(\mathrm{Y} 5)$ ), a remarkably neat result, and one of the main calculations of this paper. The requirement that (4) be soluble is satisfied if (Y7) holds, and thus if the $t_{n}^{a}$ form a Yangian. We believe the reverse implication to be true also, but it does not automatically follow, and cannot be proved without knowing the $\mathcal{O}\left(\epsilon^{2}\right)$ terms (which will depend on the quantization prescription) exactly.

Now let us examine what happens for non-zero $k$. After some calculation we find that $L(u) \rightarrow L\left(u-\frac{c}{1-u}\right)$, where $c=\epsilon k \Delta C_{2} / \lambda \hbar$, and thence

$$
L_{N}^{1}(u) L_{N}^{2}\left(u^{\prime}\right)=1 \otimes 1+T_{u-\frac{c}{1-u}} \otimes T_{u^{\prime}-\frac{c}{1-u^{\prime}}} \sum_{n=0}^{\infty} \Delta\left(t_{n}^{a}\right) j_{N \mu}^{a}+\mathcal{O}\left(\epsilon^{2}\right)
$$

up to terms of order $\epsilon$ proportional to $t^{a} \otimes t^{a}$, which commute with a group-invariant $R$-matrix.

\section{Yangian charges}

The conserved charges may now be extracted as coefficients of powers of $u$ in $T=$ $\prod_{N=-\infty}^{\infty} L_{N}$, and we find that

$$
T(u)=\exp \left(-\sum_{n=0}^{\infty} \frac{1}{u^{n+1}} \sum_{p=0}^{n}\left(\begin{array}{l}
n \\
p
\end{array}\right)(-1)^{p} Q_{p}^{a} t_{n-p}^{a}\right)+\mathcal{O}\left(\epsilon^{2}\right)
$$

where

$$
\begin{aligned}
Q_{0}^{a} & =\sum_{N=-\infty}^{\infty} j_{N 0}^{a} \\
Q_{1}^{a} & =\sum_{N=-\infty}^{\infty}\left(j_{N 1}^{a}+\frac{1}{2} f^{a b c} j_{N 0}^{b} \sum_{N^{\prime}=-\infty}^{N-1} j_{N^{\prime} 0}^{c}\right)
\end{aligned}
$$

and higher charges are defined by iteration, equivalent to the ('algebra-valued'[11]) charges produced by the usual classical procedure ${ }^{[10]}$. It follows that the charges satisfy $(\mathrm{Y} 1, \mathrm{Y} 3)$, 
whilst the coproduct can be defined as for Bernard's continuum charges or (equivalently) by examining the classical limit ${ }^{[4,12]}$, and is indeed (Y2).

Now recall ${ }^{[1,2]}$ that a Lorentz boost $L_{\theta}$ which adds $\theta$ to the rapidity of a state on which the charges are measured has the effect

$$
L_{\theta}: Q_{0}^{a} \mapsto Q_{0}^{a}, \quad Q_{1}^{a} \mapsto Q_{1}^{a}-\frac{\hbar g}{4 i \pi} \theta Q_{0}^{a}=T_{\frac{\hbar g}{4 i \pi} \theta} Q_{1}^{a} .
$$

(This is easily seen to apply in the same way to the lattice charges.) It may be checked that

$$
L_{\theta} T(u)=T\left(u-\frac{\hbar g}{4 i \pi} \theta\right) .
$$

\section{Overview}

None of these results is surprising if one takes the view ${ }^{[11]}$ that the most efficient way to construct an integrable model in this way is to take an abstract, formal $R$-matrix and represent it on combinations of auxiliary and quantum spaces as required. The monodromy matrix is then just the formal $R$-matrix evaluated on one quantum and one auxiliary space, and it is necessarily true in this approach that $T$ depends additively on $u$ and $\theta$, as in (6). The Yang-Baxter equation (YBE) on three quantum spaces gives factorization of the scattering matrix; on two quantum and one auxiliary space, conservation of conserved charges (this is well described in the literature ${ }^{[1,13]}$ ); on two auxiliary and one quantum space, the integrability condition (5); and on three auxiliary spaces, the YBE for the $R$ matrix. The underlying algebra, the Yangian, is the same whether acting on the quantum or the auxiliary space, and the four possibilities just outlined are (Y7) and (Y8) for its two manifestations. That all the results necessary for this approach can be proved in the explicit QISM-motivated construction above is rather satisfying, and should perhaps be taken as an indication that any consistent lattice quantization of an integrable 1+1-D field theory must be performed in this way. 


\section{Appendix: The Yangian algebra}

The Yangian ${ }^{[3]} \mathrm{Y}(\mathcal{A})$ of a Lie algebra $\mathcal{A}$ is generated by $I_{0}^{a}, I_{1}^{a}$ satisfying

$$
\begin{aligned}
& {\left[I_{0}^{a}, I_{0}^{b}\right]=\alpha f^{a b c} I_{0}^{c}} \\
& {\left[I_{0}^{a}, I_{1}^{b}\right]=\alpha f^{a b c} I_{1}^{c}}
\end{aligned}
$$

where $f^{a b c}$ are the structure constants of $\mathcal{A}$. The bi-algebra structure is given by the trivial co-unit

$$
\epsilon\left(I_{0}^{a}\right)=0=\epsilon\left(I_{1}^{a}\right), \quad \epsilon(1)=1
$$

and the coproduct

$$
\begin{aligned}
& \Delta\left(I_{0}^{a}\right)=1 \otimes I_{0}^{a}+I_{0}^{a} \otimes 1 \\
& \Delta\left(I_{1}^{a}\right)=1 \otimes I_{1}^{a}+I_{1}^{a} \otimes 1+\frac{\alpha^{\prime}}{2} f^{a b c} I_{0}^{c} \otimes I_{0}^{b} .
\end{aligned}
$$

Requiring that (Y2) be a homomorphism gives

$$
\frac{1}{2} f^{d[a b}\left[I_{1}^{c]}, I_{1}^{d}\right]=\alpha \alpha^{\prime 2} a_{a b c p q r} I_{0}^{(p} I_{0}^{q} I_{0}^{r)},
$$

where [] and () denote (anti-)symmetrization on the enclosed indices and

$$
a_{a b c p q r}=\frac{1}{24} f^{a p i} f^{b q j} f^{c r k} f^{i j k} .
$$

(This applies for $\mathcal{A} \neq \operatorname{sl}(2)$; for $\mathcal{A}=\operatorname{sl}(2)$ see Drinfeld.) One should think of $\mathrm{Y}(\mathcal{A})$ as being generated by a series of generators in adjoint representations of $\mathcal{A}$ at grades $0,1,2, .$. , with the $I_{0}^{a}$ and $I_{1}^{a}$ being simply the first two sets, at grades 0 and 1 respectively (and then $\alpha^{\prime}$ formally has grade 1). The condition (Y3) then gives a constraint on the construction of higher grade generators from products of $I_{1}$ s. Specifically, defining

$$
I_{p}^{a} \equiv \frac{1}{g \alpha} f^{a b c}\left[I_{1}^{c}, I_{p-1}^{b}\right]
$$

we find, applying (Y2) to (Y4), that

$\Delta\left(I_{p}^{a}\right)=I_{p}^{a} \otimes 1+1 \otimes I_{p}^{a}+\frac{\alpha^{\prime}}{2} f^{a b c}\left(I_{p-1}^{c} \otimes I_{0}^{b}+I_{p-2}^{c} \otimes I_{1}^{b}+\ldots+I_{1}^{c} \otimes I_{p-2}^{b}+I_{0}^{c} \otimes I_{p-1}^{b}\right)+\mathcal{O}\left(\alpha^{\prime 2}\right)$.

We have given $\mathrm{Y}(\mathcal{A})$ in terms of two deformation parameters $\alpha, \alpha^{\prime}$, one of which may be scaled out. In the charge algebra we have $I_{p}^{a} \equiv Q_{p}^{a}$ and $\alpha=i \hbar, \alpha^{\prime}=1$, whilst in the auxiliary Yangian we have $I_{p}^{a} \equiv t_{p}^{a}$ and $\alpha=1, \alpha^{\prime}=i \hbar$. 
To make $\mathrm{Y}(\mathcal{A})$ a Hopf algebra we also need the antipode map

$$
s\left(I_{0}^{a}\right)=-I_{0}^{a} \quad \text { and } \quad s\left(I_{1}^{a}\right)=-I_{1}^{a}+\frac{1}{2} f^{a b c} I_{0}^{b} I_{0}^{c},
$$

which is an algebra antihomomorphism. This satisfies

$$
\cdot(s \otimes 1) \sigma \circ \Delta=\cdot(1 \otimes s) \sigma \circ \Delta=0
$$

where - denotes multiplication (in the enveloping algebra) and $\sigma: x \otimes y \mapsto y \otimes x$ is the transposition operator. (This differs from the usual axiom in that we have used $\sigma \circ \Delta$ rather than $\Delta$; this has been done to maintain the conventional ordering of the currents in the quantum definition of the non-local charge.)

Further, the Yangian has an automorphism $T_{\lambda}, \lambda \in \mathcal{C}$, given by

$$
T_{\lambda}: I_{1}^{a} \mapsto I_{1}^{a}-\lambda I_{0}^{a} \quad \text { and } \quad T_{\lambda}: I_{0}^{a} \mapsto I_{0}^{a}
$$

and more generally

$$
T_{\lambda}: I_{p}^{a} \mapsto \sum_{r=0}^{p}\left(\begin{array}{l}
p \\
r
\end{array}\right)(-\lambda)^{r} I_{p-r}^{a} .
$$

There then exists a formal $R$-matrix, $\mathcal{R}(\lambda)$, with $\left(T_{\lambda_{1}} \otimes T_{\lambda_{2}}\right) \mathcal{R}(\lambda)=\mathcal{R}\left(\lambda+\lambda_{1}-\lambda_{2}\right)$, satisfying

$$
\left(1 \otimes T_{\lambda}\right) \sigma \circ \Delta(x)=\mathcal{R}(\lambda)^{-1}\left(1 \otimes T_{\lambda}\right) \Delta(x) \mathcal{R}(\lambda) \quad(x \in Y(\mathcal{A}))
$$

this $\mathcal{R}$ satisfies the Yang-Baxter equation,

$$
\mathcal{R}_{12}(\lambda) \mathcal{R}_{13}\left(\lambda+\lambda^{\prime}\right) \mathcal{R}_{23}\left(\lambda^{\prime}\right)=\mathcal{R}_{23}\left(\lambda^{\prime}\right) \mathcal{R}_{13}\left(\lambda+\lambda^{\prime}\right) \mathcal{R}_{12}(\lambda)
$$

and

$$
\mathcal{R}_{21}(\lambda) \mathcal{R}_{12}(-\lambda)=1
$$

In the auxiliary algebra $\lambda=u$, whilst in the charge algebra $\lambda=\frac{\hbar g}{4 i \pi} \theta$, this scaling being fixed by the definition of the charges or (in the exact $S$-matrix approach) by the requirement of crossing symmetry. 


\section{References}

[1] D. Bernard, Comm.Math.Phys. 137 (1991) 191.

[2] M. Lüscher, Nucl.Phys. B135 (1978) 1.

[3] V. G. Drinfeld, Sov.Math.Dokl. 32 (1985) 254, and in Proc.ICM (Berkeley,1986).

[4] N. J. MacKay, Phys.Lett. B281 (1992) 90, erratum: ibid.B308(1993)444.

[5] P. P. Kulish and N. Y. Reshetikhin, J.Sov.Math. 23 (1983) 2435.

[6] L. Faddeev, E. Sklyanin and L. Takhtajan, Theor.Math.Phys. 40 (1980) 688.

[7] P. P. Kulish and E. K. Sklyanin, Springer Physics Notes 151 (1982) 61.

[8] N. J. MacKay, Mod.Phys.Lett. A9 (1994) 2353.

[9] L. Faddeev and N. Y. Reshetikhin, Ann.Phys.(NY) 167 (1986) 227.

[10] E. Brézin, C. Itzykson, J. Zinn-Justin and J.-B. Zuber, Phys.Lett. 82B (1979) 442.

[11] H. de Vega, H. Eichenherr and J. M. Maillet, Phys.Lett. 132B (1983) 337; Nucl.Phys. B240 (1984) 377; Comm.Math.Phys. 92 (1984) 507.

[12] M. Lüscher and K. Pohlmeyer, Nucl.Phys. B137 (1978) 46.

[13] N. J. MacKay, J.Phys. 25A (1992) L1343. 